Bull. Korean Math. Soc. 50 (2013), No. 1, pp. 201-216

http://dx.doi.org/10.4134/BKMS.2013.50.1.201

\title{
CONSTRUCTION OF THE 2D RIEMANN SOLUTIONS FOR A NONSTRICTLY HYPERBOLIC CONSERVATION LAW
}

\author{
Meina Sun
}

\begin{abstract}
In this note, we consider the Riemann problem for a twodimensional nonstrictly hyperbolic system of conservation laws. Without the restriction that each jump of the initial data projects one planar elementary wave, six topologically distinct solutions are constructed by applying the generalized characteristic analysis method, in which the delta shock waves and the vacuum states appear. Moreover we demonstrate that the nature of our solutions is identical with that of solutions to the corresponding one-dimensional Cauchy problem, which provides a verification that our construction produces the correct global solutions.
\end{abstract}

\section{Introduction}

Nonlinear hyperbolic conservation laws have not only important physical background but also special interest and difficulty in mathematics. A major difficulty is that discontinuities may appear in their solutions even though the initial data are very smooth. The theory on basic one-dimensional conservation laws is fairly complete, while the analysis of multi-dimensional systems is relatively less except scalar case and some special one. Multi-dimensional conservation laws are more physical and we are interested in the global structure and the evolution of discontinuity of solutions for them.

Our main interest in this note is to construct explicitly the global solutions to the following system of conservation law

$$
\left\{\begin{array}{l}
\rho_{t}+(\rho u)_{x}+(\rho u)_{y}=0 \\
u_{t}+\left(\frac{u^{2}}{2}\right)_{x}+\left(\frac{u^{2}}{2}\right)_{y}=0
\end{array}\right.
$$

Received July 11, 2011

2010 Mathematics Subject Classification. 35L65, 35L67, 76N15.

Key words and phrases. Riemann problem, generalized characteristic analysis, delta shock wave, vacuum state.

This work is partially supported by National Natural Science Foundation of China (11271176, 10901077, 11001116), Shandong Provincial Doctoral Foundation (BS2010SF006), Shandong Provincial Natural Science Foundation (ZR2010AL012) and the Project of Shandong Provincial Higher Educational Science and Technology Program (J11LA03). 
with the Riemann initial data

$$
\left.(\rho, u)(t, x, y)\right|_{t=0}=\left(\rho_{i}, u_{i}\right) \text { for }(x, y) \text { in the } i \text { th quadrant, }
$$

where $\left(\rho_{i}, u_{i}\right), i=1,2,3,4$, are constant states.

The system (1.1) can be derived directly from the two-dimensional system of zero-pressure gas dynamics in non-conservative form [1]

$$
\left\{\begin{array}{l}
\rho_{t}+(\rho u)_{x}+(\rho v)_{y}=0, \\
u_{t}+u u_{x}+v u_{y}=0, \\
v_{t}+u v_{x}+v v_{y}=0,
\end{array}\right.
$$

by letting $u=v$, namely assuming the speeds of the $x$-axis and $y$-axis direction are equal.

Obviously (1.1) is not strictly hyperbolic for its coinciding eigenvalues. In fact, one cannot expect $\rho$ to be bounded variation and the measure should be considered. Thus we should deal with wave interactions including the delta shock wave for its resonant wave structure. About the delta shock wave solution in the multi-dimensional hyperbolic conservation laws, we can see $[4,7,9,15$, $18,20,21]$ and the reference therein.

For most of the work on the two-dimensional gas dynamics models, the initial Riemann data is restricted to satisfy the assumption that only a planar elementary wave appears at each interface of the initial data [23, 24]. In the present paper, we remove this restriction in the development of our solutions for the reason that generally an $n \times n$ system would be expected to develop $n$ waves from each initial discontinuity. Based on the generalized characteristic analysis, we solve the Riemann problem (1.1) and (1.2) analytically and six exact entropy solutions with different geometric structures are constructed globally. The solutions reveal various interactions of waves involving not only the classical waves as contact discontinuities but also the nonclassical waves as delta shock waves, meanwhile the process for the delta shock wave penetrating the vacuum state is also presented. Another contribution of this paper is that the explicit expressions for the strengths of the delta shocks are provided in details.

Moreover it can be verified that our solutions are globally unique by comparing with some one-dimensional rotated solutions of the following system of hyperbolic conservation laws

$$
\left\{\begin{array}{c}
\rho_{t}+(\rho u)_{x}=0 \\
u_{t}+\left(\frac{u^{2}}{2}\right)_{x}=0 .
\end{array}\right.
$$

Although existence and uniqueness results for the solutions to systems in one-dimensional are known, there are no such results for systems in twodimension. To our knowledge, there is no general uniqueness theory applicable to (1.1). However, under a rotation, the problem (1.1) and (1.2) can be converted into a one-dimensional Cauchy problem of (1.4) whose initial data can be described as the interacting Riemann problem [6, 10, 11, 22]. More 
precisely, the equality between the one-dimensional Cauchy solutions and the two-dimensional Riemann solutions constructed here can be derived through a mapping as in $[6,10,11,22]$, which provides a check that our construction produces the correct global solutions. The detailed construction of the onedimensional rotated solutions may refer to [17] by using the method of split delta function proposed in $[13,14]$.

The rest of the paper is organized as follows. In Section 2, we provide some basic properties of system (1.1), including the characteristics, bounded discontinuities and delta shock waves. Then we classify the Riemann problem according to the combinations of the exterior waves in Section 3. Finally, the global solutions are constructed and the strengths of the delta shocks are calculated in details in Section 4.

\section{Basic properties of system (1.1)}

In this section, we give the basic properties of system (1.1) before the construction of Riemann solutions for reader's convenience.

\subsection{Hyperbolicity}

For smooth solutions, the system (1.1) can be rewritten as

$$
U_{t}+A U_{x}+B U_{y}=0
$$

where

$$
U=\left(\begin{array}{l}
\rho \\
u
\end{array}\right), \quad A=B=\left(\begin{array}{cc}
u & \rho \\
0 & u
\end{array}\right) .
$$

The characteristic equation of $(1.1)$ in the direction $(\mu, \nu)$ with $\mu^{2}+\nu^{2}=1$ is

$$
\left|\begin{array}{cc}
\lambda-(\mu+\nu) u & -(\mu+\nu) \rho \\
0 & \lambda-(\mu+\nu) u
\end{array}\right|=0,
$$

which gives the double eigenvalue with the associated right eigenvector as

$$
\lambda=(\mu+\nu) u, \quad r=(1,0)^{T} .
$$

It follows that $\nabla \lambda \cdot r \equiv 0$. Therefore the system (1.1) is nonstrictly hyperbolic and linearly degenerate in the sense defined in $[9]$.

\subsection{Pseudo-characteristic}

Since both (1.1) and (1.2) are invariant under the self-similar transformation $(t, x, y) \rightarrow(\alpha t, \alpha x, \alpha y)$ with $\alpha>0$. As usual, we look for the self-similar solutions in the whole $(\xi, \eta)=(x / t, y / t)$ plane. The system $(1.1)$ can be reduced to the self-similar form

$$
\left\{\begin{array}{c}
-\xi \rho_{\xi}-\eta \rho_{\eta}+(\rho u)_{\xi}+(\rho u)_{\eta}=0 \\
-\xi u_{\xi}-\eta u_{\eta}+\left(\frac{u^{2}}{2}\right)_{\xi}+\left(\frac{u^{2}}{2}\right)_{\eta}=0
\end{array}\right.
$$


and (1.2) becomes the boundary value at infinity

$$
\lim _{\xi^{2}+\eta^{2} \rightarrow \infty}(\rho, u)=\left(\rho_{i}, u_{i}\right), \quad i=1,2,3,4,
$$

for $(\xi, \eta) \in i$ th quadrant.

For smooth solutions, (2.5) may be rewritten as

$$
A U_{\xi}+B U_{\eta}=0
$$

where

$$
A=\left(\begin{array}{cc}
u-\xi & \rho \\
0 & u-\xi
\end{array}\right), \quad B=\left(\begin{array}{cc}
u-\eta & \rho \\
0 & u-\eta
\end{array}\right) .
$$

Then, the characteristic equation of $(2.5)$ is

$$
\left|\begin{array}{cc}
\tilde{\lambda}(u-\xi)-(u-\eta) & (\widetilde{\lambda}-1) \rho \\
0 & \widetilde{\lambda}(u-\xi)-(u-\eta)
\end{array}\right|=0,
$$

which defines a double eigenvalue $\tilde{\lambda}=(u-\eta) /(u-\xi)$ for $(2.5)$. The corresponding right eigenvector is $r=(1,0)^{T}$, then a simple calculation leads to $\nabla \widetilde{\lambda} \cdot r \equiv 0$ which means that $(2.5)$ is linear degenerate.

The integral curve defined by $d \eta / d \xi=\widetilde{\lambda}$ is called a characteristic curve of (2.5), denoted by $\Gamma$. $\widetilde{\lambda}$ is also called a pseudo-characteristic of (1.1). A characteristic curve $\eta=\eta(\xi)$ of $(2.5)$ in the $(\xi, \eta)$ plane corresponds to a characteristic surface $y=t \eta(x / t)$ of $(1.1)$ in the $(t, x, y)$ space with the normal direction $(-\lambda, \mu, \nu)=(\xi \widetilde{\lambda}-\eta,-\widetilde{\lambda}, 1)$.

The singularity points for $\Gamma$ are $(\xi, \eta)=(u, u)$ which lie on the line $\xi=\eta$. Motivated by the increase of time, we stipulate the direction of the curve $\Gamma$ from the point on them to the singularity point $(\xi, \eta)=(u, u)$. For the details, refer to Chapter one in [9].

\subsection{Bounded discontinuities}

Let $\eta=\eta(\xi)$ be a smooth discontinuity of a bounded discontinuous solution in the $(\xi, \eta)$ plane, i.e., $y=t \eta(x / t)$ is a discontinuity surface in the $(t, x, y)$ space. The normal direction of the surface is $(\eta-\xi \sigma, \sigma,-1)$ with $\sigma=d \eta / d \xi$. Suppose the limit states on two sides of the discontinuity are $\left(\rho_{+}, u_{+}\right)$and $\left(\rho_{-}, u_{-}\right)$respectively. Then the following Rankine-Hugoniot relation should be true

$$
\left\{\begin{array}{l}
-(\eta-\xi \sigma)[\rho]-\sigma[\rho u]+[\rho u]=0, \\
-(\eta-\xi \sigma)[u]-\sigma\left[\frac{u^{2}}{2}\right]+\left[\frac{u^{2}}{2}\right]=0,
\end{array}\right.
$$

where $[u]=u_{+}-u_{-}$denotes the jump of $u$ across discontinuity, etc.

Solving (2.10), we obtain

$$
\frac{d \eta}{d \xi}=\sigma=\frac{\eta-u_{+}}{\xi-u_{+}}=\frac{\eta-u_{-}}{\xi-u_{-}}, \quad u_{+}=u_{-}
$$


We call the discontinuity defined by (2.11) a contact discontinuity, denoted by $J$, which coincides with the characteristic lines for both sides. Similarly to $\Gamma$, we orient the integral curve of $d \eta / d \xi=\sigma$ to point towards the singularity point $(\xi, \eta)=\left(u_{+}, u_{+}\right)$which also locates on the line $\xi=\eta$.

\subsection{Delta shock wave and vacuum state}

Solutions of (2.5) and (2.6) must be one-dimensional planar waves besides constant states in the neighborhood of infinity in the $(\xi, \eta)$ plane, that is, for sufficiently large $(\xi, \eta),(1.1)$ and $(1.2)$ can be determined as a one-dimensional problem. According to the Riemann solutions to (1.1) in one-dimension (see [17]), the delta shock wave denoted by $S_{\delta}$ must appear to connect the states $\left(\rho_{i}, u_{i}\right)$ and $\left(\rho_{j}, u_{j}\right)$ if $\Phi_{i j}=u_{i}-u_{j}>0$, where $i j \in\{21,32,34,41\}$. Therefore, the definition of a measure solution to (1.1) and the generalized RankineHugoniot relation in three-dimension should be given like as in $[2,8,9,16,19]$.

Definition 1. The three-dimensional weighted $\delta$-measure $w(t, s) \delta_{S}$ supported on a smooth surface $S: x=x(t, s), y=y(t, s)$ can be defined by

$$
\left\langle w(t, s) \delta_{S}, \phi\right\rangle=\int_{0}^{+\infty} \int_{0}^{+\infty} w(t, s) \phi(t, x(t, s), y(t, s)) d s d t
$$

for any test function $\phi(t, x, y) \in C_{0}^{\infty}\left([0,+\infty) \times \mathbb{R}^{2}\right)$.

Let a discontinuity surface $S$ be smooth and be parameterized as $S: x=$ $x(t, s), y=y(t, s)$ with the parameter $s \in[0,+\infty)$, which divides the $(t \geq$ $0, x, y)$ space into two infinite parts $\Omega_{-}$and $\Omega_{+}$. The positiveness of $s$ here is a purely technical consideration and the strength $w(t, s)$ of the delta shock wave $S_{\delta}$ depends on the choice of $s$. Let $n$ be the normal of the surface $S$ chosen to point from $\Omega_{-}$to $\Omega_{+}$.

With the above definition, we seek the delta shock type solution in the following form

$$
(\rho, u)(t, x, y)= \begin{cases}\left(\rho_{-}, u_{-}\right), & (t, x, y) \in \Omega_{-}, \\ \left(w(t, s) \delta(t, x-x(t, s), y-y(t, s)), u_{\delta}(t, s)\right), & (t, x, y) \in S, \\ \left(\rho_{+}, u_{+}\right), & (t, x, y) \in \Omega_{+},\end{cases}
$$

where $w(t, s) \in C^{1}([0,+\infty) \times[0,+\infty))$ is the strength of the delta shock wave, $u_{\delta}(t, s)$ is the valuation of $u$ on the surface $S$ of the delta shock wave, and $\delta$ is the standard Dirac delta function supported on the surface $S$.

Definition 2. The delta shock type solution (2.13) satisfies (1.1) in the sense of distribution when the following equalities

$$
\left\{\begin{array}{l}
\left\langle\rho, \phi_{t}\right\rangle+\left\langle\rho u, \phi_{x}\right\rangle+\left\langle\rho u, \phi_{y}\right\rangle=0 \\
\left\langle u, \phi_{t}\right\rangle+\left\langle\frac{u^{2}}{2}, \phi_{x}\right\rangle+\left\langle\frac{u^{2}}{2}, \phi_{y}\right\rangle=0
\end{array}\right.
$$


hold for all test function $\phi(t, x, y) \in C_{0}^{\infty}\left([0,+\infty) \times \mathbb{R}^{2}\right)$, in which

$$
\begin{aligned}
\langle\rho, \phi\rangle & =\int_{\Omega_{-}} \rho_{-} \phi d x d y d t+\int_{\Omega_{+}} \rho_{+} \phi d x d y d t+\left\langle w \delta_{S}, \phi\right\rangle \\
\langle\rho u, \phi\rangle & =\int_{\Omega_{-}} \rho_{-} u_{-} \phi d x d y d t+\int_{\Omega_{+}} \rho_{+} u_{+} \phi d x d y d t+\left\langle u_{\delta} w \delta_{S}, \phi\right\rangle .
\end{aligned}
$$

It can be claimed that (2.13) is a solution of (1.1) in the sense of distribution if the following generalized Rankine-Hugoniot condition is satisfied

$$
\left\{\begin{array}{l}
\frac{\partial x}{\partial t}=\frac{\partial y}{\partial t}=u_{\delta}(t, s), \\
\frac{\partial w(t, s)}{\partial t}=([\rho],[\rho u],[\rho u]) \cdot\left(n_{t}, n_{x}, n_{y}\right), \\
0=\left([u],\left[\frac{u^{2}}{2}\right],\left[\frac{u^{2}}{2}\right]\right) \cdot\left(n_{t}, n_{x}, n_{y}\right),
\end{array}\right.
$$

in which $[u]=u_{-}-u_{+}$denotes the jump of $u$ across the discontinuity surface $S$ etc and the normal of $S$ can be calculated by

$$
\left(n_{t}, n_{x}, n_{y}\right)=\left(u_{\delta}\left(\frac{\partial y}{\partial s}-\frac{\partial x}{\partial s}\right),-\frac{\partial y}{\partial s}, \frac{\partial x}{\partial s}\right) .
$$

The generalized Rankine-Hugoniot relation of delta shock wave reflects the exact relationship among the location, propagation speed, weight and the assignment of $u_{\delta}$ on its discontinuity. Moreover we can supplement the geometrical entropy condition for the delta shock wave as follows:

$$
u_{-}>u_{\delta}>u_{+} \text {if } n_{x}+n_{y}>0 \quad \text { or } \quad u_{-}<u_{\delta}<u_{+} \text {if } n_{x}+n_{y}<0
$$

which means that the characteristic lines on both sides of the delta shock are incoming in the $(t, x, y)$ space.

To apply the method of generalized characteristic analysis, the expression for the delta shock in the $(\xi, \eta)$ plane should be clarified. Assume $\eta=\eta(\xi)$ with $\tau=d \eta / d \xi$ is a discontinuity in the $(\xi, \eta)$ plane, which is a discontinuity surface $y=t \eta(x / t)$ with normal direction $(\eta-\xi \tau, \tau,-1)$ in the $(t, x, y)$ space. So the third equation in (2.15) has the form $[u](\eta-\xi \tau)+\tau\left[u^{2} / 2\right]-\left[u^{2} / 2\right]=0$, which gives

$$
\tau=\frac{d \eta}{d \xi}=\frac{\eta-\left(u_{+}+u_{-}\right) / 2}{\xi-\left(u_{+}+u_{-}\right) / 2} .
$$

We also orient the integral curve of $d \eta / d \xi=\tau$ to point towards the singularity point $(\xi, \eta)=\left(\left(u_{+}+u_{-}\right) / 2,\left(u_{+}+u_{-}\right) / 2\right)$ on the line $\xi=\eta$.

Finally, let us turn our attentions to the vacuum state defined like in [12] that a vacuum state is any portion in the $(t, x, y)$ space in which the density $\rho \equiv 0$ is achieved. We can see that the Riemann solutions do involve the vacuum state for certain Riemann data. In order to encounter the interactions of the delta shock waves with the vacuum states, we adopt the idea proposed by Liu and Smoller [12] when they considered the vacuum problem for the isentropic 
gas dynamic equations, and then approximate them by a set of small nonadmissible shocks like as the front tracking algorithm [3]. In a word, they made a distinction between two vacuum states with different (fake) velocities.

\subsection{Planar elementary waves}

By initial condition (2.6), in the neighborhood of infinity in the $(\xi, \eta)$ plane, the discontinuities (contact discontinuities or delta shock waves) must be lines parallel to the $\xi$-axis or $\eta$-axis. These waves are called exterior waves and they are one-dimensional planar elementary waves.

We can solve the problem (2.5) and (2.6) in the neighborhood of infinity to obtain the exterior solutions which consist of one-dimensional planar elementary waves. For example, to connect the states $\left(\rho_{1}, u_{1}\right)$ and $\left(\rho_{2}, u_{2}\right)$ of $(2.6)$, we have the following possibilities:

(i) a contact discontinuity: $\xi=u_{1}=u_{2}$ for $u_{1}=u_{2}$;

(ii) two contact discontinuities: $\xi=u_{2}$ and $\xi=u_{1}$ between which lies the vacuum state $\rho=0$ for $u_{2}<u_{1}$;

(iii) a delta shock wave: $\xi=u_{1}+u_{2}$ for $u_{2}>u_{1}$.

\section{Classification}

Under the transformation

$$
\bar{\xi}=(x+y) / 2, \quad \bar{\eta}=(y-x) / 2,
$$

which is a rotation of $\pi / 4=\tan ^{-1}(1)$ combined with a dilation by a factor of $\sqrt{2}$, the system (1.1) changes into

$$
\left\{\begin{array}{c}
\rho_{t}+(\rho u)_{\bar{\xi}}=0 \\
u_{t}+\left(\frac{u^{2}}{2}\right)_{\bar{\xi}}=0
\end{array}\right.
$$

which is exactly the system (1.4). So the solutions can be obtained in each $(\bar{\xi}, \bar{\eta}=$ const, $t)$ plane independent of other $\bar{\eta}$. Especially, the solutions in the $\bar{\eta}>0(y>x)$ half-plane can be constructed independently of those in the $\bar{\eta}<0(y<x)$ half-plane. In fact, the catalog of solutions in the two half-planes is the same. Therefore our attention can be restricted to the solutions in the $\bar{\eta}>0$ plane, which can be connected with those in the $\bar{\eta}<0$ half-plane in a consistent manner along the $\bar{\eta}=0(y=x)$ axis. Turning to the problem of initial data specified as (1.2), there is no need to consider data in quadrant 4. See Fig. 3.1. Here and below, the symbol (i) is used to stand for the state $\left(\rho_{i}, u_{i}\right), i=1,2,3,4$.

Recalling the Riemann solutions to (1.1) in one-dimension [17], we know that, in the neighborhood of infinity in the $(\xi, \eta)$ plane, a delta shock wave, a contact discontinuity, or a combination of two contact discontinuities and the vacuum state emits from each interface of the initial data (1.2). Then according to the exterior waves propagating in the $y$-direction and in the $x$-direction, 
we find that there exist six different combinations which lead to topologically distinct solutions.

The combinations are as follows:

1. $S_{\delta}$ and $J+$ Vacuum $+J$,

2. $S_{\delta}$ and $J$,

3. $S_{\delta}$ and $S_{\delta}$,

4. $J+$ Vacuum $+J$ and $J+$ Vacuum $+J$,

5. $J+\operatorname{Vacuum}+J$ and $J$,

6. $J$ and $J$.

The discussions for all other possible combinations, that is $J+$ Vacuum $+J$ and $S_{\delta}, J$ and $S_{\delta}, J$ and $J+$ Vacuum $+J$, can be reduced to those for the above six cases. For example, the combination $J+$ Vacuum $+J$ and $S_{\delta}$, which means $J+$ Vacuum $+J$ propagating in the $y$-direction and $S_{\delta}$ propagating in the $x$-direction can be treated in the same way as Case 1 .

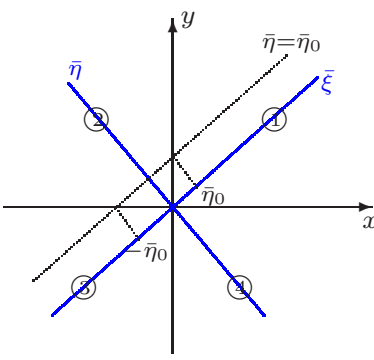

(a)

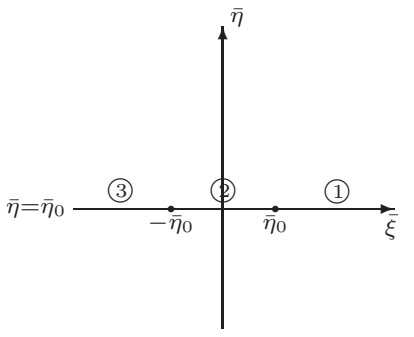

(b)

Fig. 3.1: (a) the initial Riemann data and the rotated $(\bar{\xi}, \bar{\eta})$ coordinate system; (b) the initial one-dimensional Cauchy data along a line $\bar{\eta}=\bar{\eta}_{0}$.

\section{Construction of solutions}

What we need to do in the following is to extend the exterior waves in the neighborhood of infinity inwards to construct our global Riemann solutions. We will deal with this problem case by case according to the above classification and our results can be summarized in the following.

Theorem 1. The Riemann solutions of (1.1) and (1.2) can be explicitly constructed by a two-dimensional constructive technique, i.e., the method of generalized characteristic analysis. Comparing the solutions that we construct with the solutions for the one-dimensional Cauchy problem (3.1) whose initial data can be described as interacting Riemann problem demonstrates the equality of the solutions, so our construction provides the correct global solutions.

For simplicity, in the following figures, we use the symbols $(i)$ and $(\overline{i+j})$ to represent the points $(\xi, \eta)=\left(u_{i}, u_{i}\right)$ and $(\xi, \eta)=\left(\left(u_{i}+u_{j}\right) / 2,\left(u_{i}+u_{j}\right) / 2\right)$ respectively. 
In the following Cases 4.1-4.3, the exterior wave connecting states $\left(u_{2}, \rho_{2}\right)$ and $\left(u_{1}, \rho_{1}\right)$ is a delta shock wave denoted by $S_{\delta}^{1}: \xi=x / t=\left(u_{1}+u_{2}\right) / 2$ in the neighborhood of infinity in the $(\xi, \eta)$ plane, so that $u_{2}>u_{1}$ must hold.

To keep the positiveness of $s$, the surface of $S_{\delta}^{1}$ can be parameterized as

$$
x=\frac{u_{1}+u_{2}}{2} t, \quad y=\frac{u_{1}+u_{2}}{2} t-s,
$$

which, combining with (2.15) and (2.16), gives that

$$
u_{\delta}=\frac{u_{1}+u_{2}}{2}, \quad\left(n_{t}, n_{x}, n_{y}\right)=\left(-\frac{u_{1}+u_{2}}{2}, 1,0\right) .
$$

It follows from (2.15) that

$$
\begin{aligned}
\frac{\partial w}{\partial t} & =\left(\rho_{2}-\rho_{1}, \rho_{2} u_{2}-\rho_{1} u_{1}, \rho_{2} u_{2}-\rho_{1} u_{1}\right) \cdot\left(-\frac{u_{1}+u_{2}}{2}, 1,0\right) \\
& =\frac{\left(u_{2}-u_{1}\right)\left(\rho_{1}+\rho_{2}\right)}{2} .
\end{aligned}
$$

By taking into account the fact $w(0, s)=0$, we obtain the strength

$$
w(t, s)=\frac{\left(u_{2}-u_{1}\right)\left(\rho_{1}+\rho_{2}\right)}{2} t
$$

which is linearly increasing with the time $t$.

Case 4.1. $S_{\delta}$ and $J+$ Vacuum $+J$.

We first present the solution obtained by the generalized characteristic analysis method, which developed from the classical characteristic method in timespace and the method of analysis in phase space. This method is a reasonable combination of the self-similar plane with the phase space and it enables us to get an explicit construction of Riemann solutions in a quite simple and straightforward way [9].

For this case, the initial data must satisfy $u_{2}>u_{1}$ and $u_{2}>u_{3}$. So there are two possible subcases: $u_{3} \leq u_{1}<u_{2}$ and $u_{1}<u_{3}<u_{2}$.

As shown in Fig. 4.1, the transition from $\left(u_{2}, \rho_{2}\right)$ to $\left(u_{3}, \rho_{3}\right)$ results in two contact discontinuities $J_{1}: \eta=u_{2}$ and $J_{2}: \eta=u_{3}$, between which is the vacuum state $\rho=0$.

Obviously, $S_{\delta}^{1}$ is unable to arrive at its singularity point $(\xi, \eta)=\left(\left(u_{1}+\right.\right.$ $\left.\left.u_{2}\right) / 2,\left(u_{1}+u_{2}\right) / 2\right)$ for the reason that it must interact with the contact discontinuity $J_{1}$ at the point $\left(\xi_{0}, \eta_{0}\right)=\left(\left(u_{1}+u_{2}\right) / 2, u_{2}\right)$. At the point $\left(\xi_{0}, \eta_{0}\right)$, the states $\left(\rho_{1}, u_{1}\right)$ and $\left(0, u_{2}\right)$ should be connected, where $u_{2}$ is a fake velocity for the vacuum state. This gives rise to a new delta shock wave denoted by $S_{\delta}^{2}: \eta=\eta(\xi)$ which will penetrate the vacuum state and has a varying speed expressed as

$$
\left\{\begin{array}{l}
\frac{d \eta}{d \xi}=\frac{\eta-\left(u+u_{1}\right) / 2}{\xi-\left(u+u_{1}\right) / 2} \\
\eta=u \\
\left(\xi_{0}, \eta_{0}\right)=\left(\left(u_{1}+u_{2}\right) / 2, u_{2}\right)
\end{array}\right.
$$


where $u \in\left[u_{1}, u_{2}\right]$ for $u_{1} \geq u_{3}$, whereas $u \in\left[u_{3}, u_{2}\right]$ for $u_{3}>u_{1}$.

From this equation, we find that the tangent line of this discontinuity always points to the singularity points $(\xi, \eta)=\left(\left(u+u_{1}\right) / 2,\left(u+u_{1}\right) / 2\right)$, where $u$ varies from $u_{2}$ to $u_{1}$ or $u_{3}$. Therefore the integral curve of (4.4) is convex, which is concluded as follows.

Lemma 2. The integral curve of (4.4) denoted by $\eta=\eta(\xi)$ is convex, i.e., $\frac{d^{2} \eta}{d \xi^{2}}>0$, and it always lies above the line $\eta=\xi$.

Proof. In fact, substituting $u=\eta$ into the first equation of (4.4) yields

$$
\frac{d \eta}{d \xi}=\frac{\eta-u_{1}}{2 \xi-\eta-u_{1}}
$$

then noticing the initial condition $\left(\xi_{0}, \eta_{0}\right)=\left(\left(u_{1}+u_{2}\right) / 2, u_{2}\right)$, we get

$$
\xi=\eta-\frac{\left(\eta-u_{1}\right)^{2}}{2\left(u_{2}-u_{1}\right)}<\eta,
$$

which proves our claim that $\eta=\eta(\xi)$ lies above the line $\eta=\xi$.

Using (4.6), we can simplify (4.5) as

$$
\frac{d \eta}{d \xi}=\frac{u_{2}-u_{1}}{u_{2}-\eta}
$$

Then differentiating this expression with respect to $\xi$ and noting that $\eta=u \leq$ $u_{2}$, we obtain

$$
\frac{d^{2} \eta}{d \xi^{2}}=\frac{\left(u_{2}-u_{1}\right)^{2}}{\left(u_{2}-\eta\right)^{3}}>0,
$$

so that the curve $\eta=\eta(\xi)$ is convex.

Now we give the explicit expression for the delta shock wave $S_{\delta}^{2}$ crossing the vacuum state, which is described in the following lemma.

Lemma 3. The strength $w(t, s)$ and $u_{\delta}(t, s)$ for the delta shock $S_{\delta}^{2}$ penetrating the vacuum state are respectively $w(t, s)=\rho_{1} \sqrt{2\left(u_{2}-u_{1}\right) s t}$ and $u_{\delta}(t, s)=$ $u_{1}+\sqrt{\frac{\left(u_{2}-u_{1}\right) s}{2 t}}$, where the parameter $s=y-x>0$.

Proof. Transfer (4.6) into the following form in the $(t, x, y)$ space as

$$
\frac{y-x}{t}=\frac{1}{2\left(u_{2}-u_{1}\right)}\left(\frac{y}{t}-u_{1}\right)^{2} .
$$

Introducing the parameter $s=y-x$ and noting $\eta \geq u_{1},(4.7)$ can be expressed as

$$
\left\{\begin{array}{l}
x=u_{1} t+\sqrt{2\left(u_{2}-u_{1}\right) s t}-s, \\
y=u_{1} t+\sqrt{2\left(u_{2}-u_{1}\right) s t} .
\end{array}\right.
$$

From (4.8), it can be derived that

$$
u_{\delta}=\frac{\partial x}{\partial t}=\frac{\partial y}{\partial t}=u_{1}+\sqrt{\frac{\left(u_{2}-u_{1}\right) s}{2 t}},
$$




$$
\frac{\partial y}{\partial s}=\sqrt{\frac{\left(u_{2}-u_{1}\right) t}{2 s}}, \quad \frac{\partial x}{\partial s}=\frac{\partial y}{\partial s}-1
$$

which, together with (2.16), yield

(4.11) $\left(n_{t}, n_{x}, n_{y}\right)=\left(u_{1}+\sqrt{\frac{\left(u_{2}-u_{1}\right) s}{2 t}},-\sqrt{\frac{\left(u_{2}-u_{1}\right) t}{2 s}},-1+\sqrt{\frac{\left(u_{2}-u_{1}\right) t}{2 s}}\right)$.

Substituting (4.11) into the second equation in (2.15), we obtain that

$$
\frac{\partial w}{\partial t}=\rho_{1} \sqrt{\frac{\left(u_{2}-u_{1}\right) s}{2 t}}
$$

then with $w(0, s)=0$ in mind, we have

$$
w(t, s)=\rho_{1} \sqrt{2\left(u_{2}-u_{1}\right) s t} .
$$

Furthermore, for $u_{1} \geq u_{3}$, we find that the delta shock wave can not penetrate over the vacuum state and it vanishes tangentially to the point $(\xi, \eta)=\left(u_{1}, u_{1}\right)$. See (a) in Fig. 4.1.

While for $u_{1}<u_{3}$, the whole vacuum state will be canceled and the ending point for the process of the canceling is $\left(\xi_{1}, u_{3}\right)$, where $\xi_{1}$ can be obtained by substituting $\eta=u_{3}$ into (4.6). Thereafter the delta shock wave reverts to a planar wave $S_{\delta}^{3}$ :

$$
\eta=\frac{u_{1}-u_{2}}{u_{3}-u_{2}} \xi+\frac{u_{3}^{2}-u_{1}^{2}}{2\left(u_{3}-u_{2}\right)},
$$

which has $\left(\rho_{1}, u_{1}\right)$ and $\left(\rho_{3}, u_{3}\right)$ as the limit states on two sides until it stops at its singularity point $(\xi, \eta)=\left(\left(u_{1}+u_{3}\right) / 2,\left(u_{1}+u_{3}\right) / 2\right)$. With the parameter $s \in[0,+\infty),(4.14)$ can be parameterized as

$$
\left\{\begin{array}{l}
x=\frac{u_{1}+u_{3}}{2} t+s \\
y=\frac{u_{1}+u_{3}}{2} t+\frac{u_{1}-u_{2}}{u_{3}-u_{2}} s
\end{array}\right.
$$

By a similar computation as above, it can be obtained that

$$
u_{\delta}=\frac{u_{1}+u_{3}}{2}, \quad \frac{\partial x}{\partial s}=1, \quad \frac{\partial y}{\partial s}=\frac{u_{1}-u_{2}}{u_{3}-u_{2}},
$$

then by (2.16), we get

$$
\left(n_{t}, n_{x}, n_{y}\right)=\left(\frac{u_{1}^{2}-u_{3}^{2}}{2\left(u_{3}-u_{2}\right)},-\frac{u_{1}-u_{2}}{u_{3}-u_{2}}, 1\right),
$$

from which and (2.15) combining with the initial condition $w(0, s)=0$, we have

$$
w(t, s)=\frac{\left(u_{1}-u_{3}\right)^{2}\left(\rho_{1}+\rho_{3}\right)}{2\left(u_{2}-u_{3}\right)} t .
$$

The global solution is illustrated in (b) of Fig. 4.1.

As stated in last section, the Riemann problem (1.1) and (1.2) can be rotated into a one-dimensional Cauchy problem. On the line $\bar{\eta}=\bar{\eta}_{0}$, the initial data discontinuities are positioned at $\bar{\xi}= \pm \bar{\eta}_{0}$, see Fig. 3.1. The details for the 
construction of the one-dimensional rotated solutions may refer to [17] by using the method of split delta function $[13,14]$.

By comparing with the rotated one-dimensional solution, it can be verified that the above constructed two-dimensional solution is correct. We regard Fig. 4.1 as a snapshot of the solution in the $t=1, x, y$ plane. Then a mapping between the solutions in Figs. 4.1 and 4.2 can be formally derived $[6,10,11]$. In fact, a cut through the solution along the line $y=x+2 \bar{\eta}_{0}$ in Fig. 4.1 is identical to that along $t=1$ in Fig. 4.2. For any $\bar{\eta}<\bar{\eta}_{0}$ (resp. $\bar{\eta}>\bar{\eta}_{0}$ ), a cut along the line $y=x+2 \bar{\eta}$ in Fig. 4.1 corresponds to that along some $t>1$ (resp. $t<1$ ) in Fig. 4.2. As $\bar{\eta} \rightarrow 0$ (resp. $\bar{\eta} \rightarrow \infty$ ), the corresponding value of $t$ tends to infinity (resp. zero). Such a comparison of qualitative features between these two figures determines that the nature of the two solutions is identical.

For brevity, we do not show the one-dimensional rotated solutions below which are identical with the two-dimensional solutions that we construct.

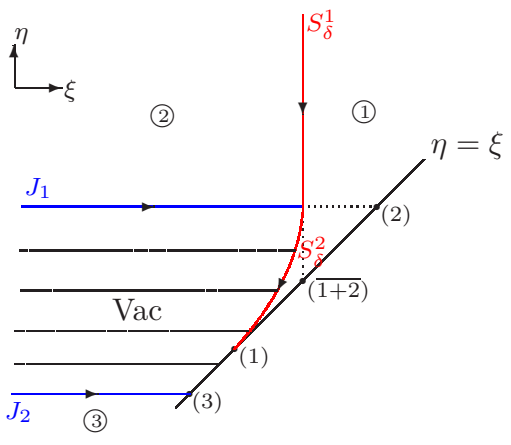

(a.) $u_{3} \leq u_{1}<u_{2}$

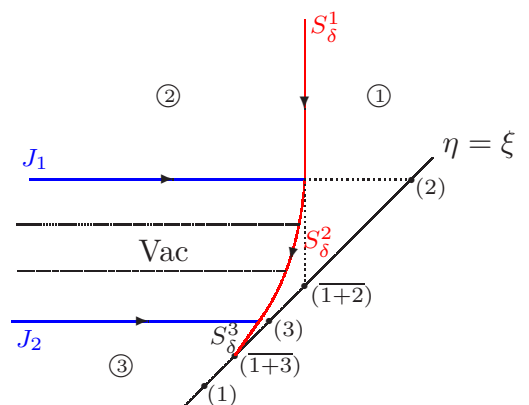

(b.) $u_{1}<u_{3}<u_{2}$

Fig. 4.1. Direct two-dimensional construction of the solution in the $(\xi, \eta)$ plane.

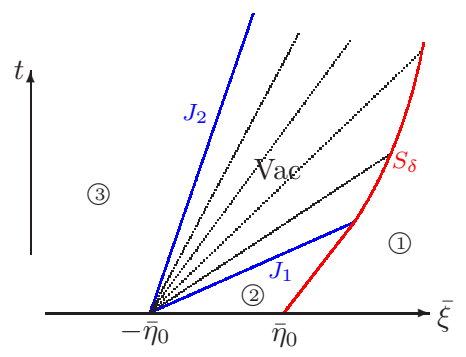

(a.) $u_{3} \leq u_{1}<u_{2}$

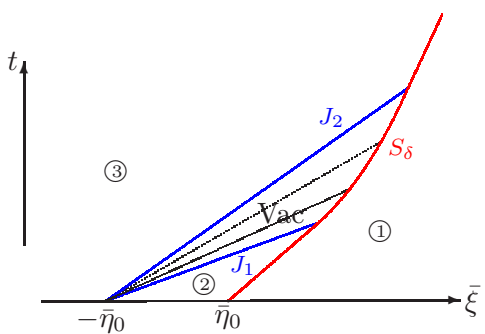

(b.) $u_{1}<u_{3}<u_{2}$

Fig. 4.2. The rotated one-dimensional solution in the $\left(t, \bar{\xi}, \bar{\eta}_{0}\right)$ plane.

Case 4.2. $S_{\delta}$ and $J$. 
This case happens if and only if $u_{3}=u_{2}>u_{1}$. The exterior wave separating the states $\left(\rho_{2}, u_{2}\right)$ and $\left(\rho_{3}, u_{3}\right)$ is a contact discontinuity $J: \eta=u_{2}=u_{3}$.

The two exterior waves $J$ and $S_{\delta}^{1}$ begin to interact at the point $\left(\xi_{0}, \eta_{0}\right)=$ $\left(\left(u_{1}+u_{2}\right) / 2, u_{3}\right)$, where the states $\left(\rho_{1}, u_{1}\right)$ and $\left(\rho_{3}, u_{3}\right)$ should be connected. This interaction gives rise to a new planar delta shock wave $S_{\delta}^{2}$ which has the same propagating direction as before, namely, $S_{\delta}^{2}: \xi=\left(u_{1}+u_{2}\right) / 2$. However, its strength occurs to change due to the difference between $\rho_{2}$ and $\rho_{3}$. In fact, in view of (2.15), we have

$$
\begin{aligned}
\frac{\partial w}{\partial t} & =\left(\rho_{3}-\rho_{1}, \rho_{3} u_{3}-\rho_{1} u_{1}, \rho_{3} u_{3}-\rho_{1} u_{1}\right) \cdot\left(-\frac{u_{1}+u_{3}}{2}, 1,0\right) \\
& =\frac{\left(u_{3}-u_{1}\right)\left(\rho_{1}+\rho_{3}\right)}{2},
\end{aligned}
$$

which, together with $w(0, s)=0$, gives

$$
w(t, s)=\frac{\left(u_{3}-u_{1}\right)\left(\rho_{1}+\rho_{3}\right)}{2} .
$$

We picture the configuration of the solution in (a) of Fig. 4.3.

Case 4.3. $S_{\delta}$ and $S_{\delta}$.

The occurrence of this case depends on the condition $u_{3}>u_{2}>u_{1}$. The exterior wave $S_{\delta}^{2}: \eta=y / t=\left(u_{2}+u_{3}\right) / 2$ appears to connect the states $\left(\rho_{2}, u_{2}\right)$ and $\left(\rho_{3}, u_{3}\right)$.

By letting $s=y-x>0$, we obtain the equations for $S_{\delta}^{2}$ in the parametric form:

$$
x=\frac{u_{2}+u_{3}}{2} t+s, \quad y=\frac{u_{2}+u_{3}}{2} t .
$$

Applying this to (2.15) and (2.16) gives

$$
u_{\delta}=\frac{u_{2}+u_{3}}{2}, \quad\left(n_{t}, n_{x}, n_{y}\right)=\left(-\frac{u_{2}+u_{3}}{2}, 0,1\right) .
$$

Then using (2.15) and $w(0, s)=0$, we obtain

$$
w(t, s)=\frac{\left(u_{3}-u_{2}\right)\left(\rho_{3}+\rho_{2}\right)}{2} t
$$

The collision of the exterior delta shocks $S_{\delta}^{1}$ and $S_{\delta}^{2}$ happens at the point $\left(\xi_{0}, \eta_{0}\right)=\left(\left(u_{1}+u_{2}\right) / 2,\left(u_{2}+u_{3}\right) / 2\right)$ where the two delta shocks coalesce into a new delta shock $S_{\delta}^{3}$, which moves along the line satisfying (4.14) and ends at the point $\left(\left(u_{1}+u_{3}\right) / 2,\left(u_{1}+u_{3}\right) / 2\right)$ in the $(\xi, \eta)$ plane. The strength $w(t, s)$ and the velocity $u_{\delta}$ for $S_{\delta}^{3}$ can be derived in the same way as those for $S_{\delta}^{3}$ in Case 4.1, and we get

$$
u_{\delta}=\frac{u_{1}+u_{3}}{2}, \quad w(t, s)=\frac{\left(u_{1}-u_{3}\right)^{2}\left(\rho_{1}+\rho_{3}\right)}{2\left(u_{3}-u_{2}\right)} t .
$$

See (b) in Fig. 4.3. 


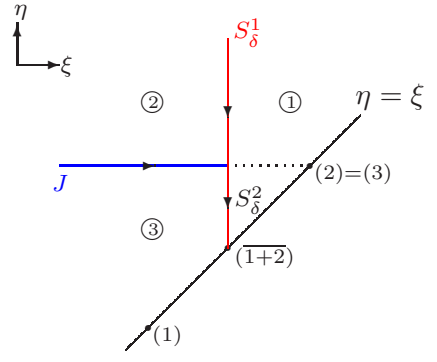

(a) $u_{2}=u_{3}>u_{1}$

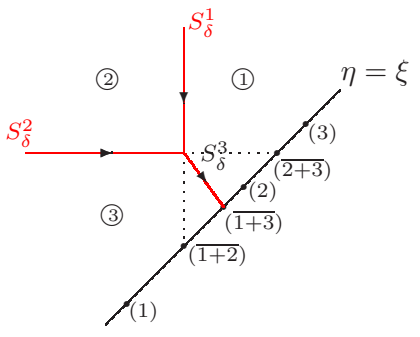

(b) $u_{3}>u_{2}>u_{1}$

Fig. 4.3

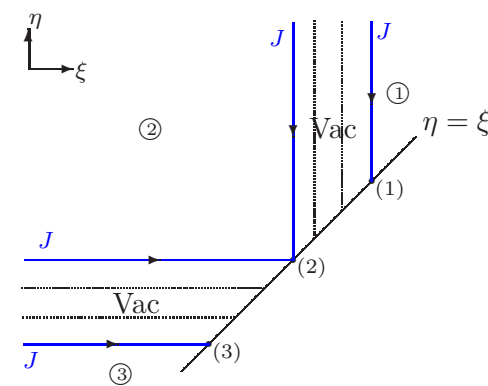

(a) $u_{3}<u_{2}<u_{1}$

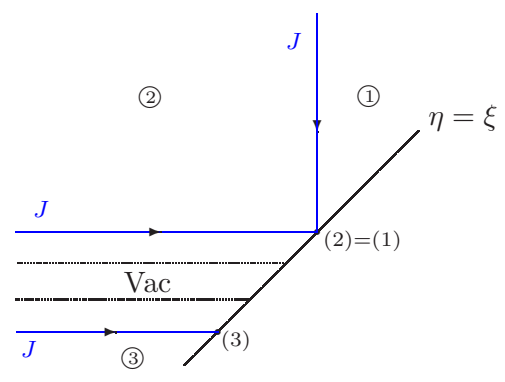

(b) $u_{3}<u_{2}=u_{1}$

Fig. 4.4

Case 4.4. The solutions not involving $S_{\delta}$.

For the remaining cases, the delta shock waves do not appear in the solutions. The initial data have the ordering: $u_{3} \leq u_{2} \leq u_{1}$ under which there are no interactions of waves. The contact discontinuities are able to go straight until reach their singularity points lying on the line $\eta=\xi$. The solutions for part of the cases are shown in Fig. 4.4.

Up to now, we have finished constructing the global structure of Riemann solutions for (1.1) completely in all the cases. The formation and evolution of singularities in the Riemann solutions are analyzed in details, which is a major difficulty in solving hyperbolic systems of conservation laws. It is remarkable that the Riemann solutions of (1.1) and (1.2) contain two extreme situations, namely one is the delta shock wave $S_{\delta}$ which is the singular solution including a delta function singularity for $\rho$ and the other is the vacuum state between two contact discontinuities. Thus, it is important to study the interaction of the delta shock wave and the vacuum state and it also serves as the foundation to construct the global Riemann solution of (1.1) and (1.2). 


\section{References}

[1] S. Albeverio and V. M. Shelkovich, On the delta-shock front, in: Analytical Approaches to Multidimensional Balance Laws (Ed. O.S.Rozanova), pp.45-88, Nova Science Publishers, 2006.

[2] F. Bouchut, On zero pressure gas dynamics, in: Advances in kinetic theory and computing, 171-190, Ser. Adv. Math. Appl. Sci., 22, World Sci. Publ., River Edge, NJ, 1994.

[3] A. Bressan, Hyperbolic Systems of Conservation Laws: The One-dimensional Cauchy Problem, Oxford Lecture Ser. Math. Appl., vol. 20, Oxford University Press, Oxford, 2000.

[4] L. Guo, W. Sheng, and T. Zhang, The Two-dimensional Riemann problem for isentropic Chaplygin gas dynamic system, Commun. Pure Appl. Anal. 9 (2010), no. 2, 431-458.

[5] F. Huang and X. Yang, The two-dimensional Riemann problem for a class of systems of hyperbolic conservation law equations, Acta Math. Appl. Sinica 21 (1998), no. 2, 193-205.

[6] W. Hwang and W. B. Lindquist, The 2-dimensional Riemann problem for a $2 \times 2$ hyperbolic law, (I) Isotropic media, (II) Anisotropic media, SIAM J. Math. Anal. 34 (2002), no. 2, 341-358; SIAM J. Math. Anal. 34 (2002), no. 2, 359-384.

[7] G. Lai, W. Sheng, and Y. Zheng, Simple waves and pressure delta waves for a Chaplygin gas in multi-dimensions, Discrete Contin. Dyn. Syst. 31 (2011), no. 2, 489-523.

[8] P. G. LeFloch, An existenceand uniqueness result for two nonstrictly hyperbolic systems, Nonlinear Evolution Equations that change Type, IMA Vol. Math. Appl. 27 ed B. Keyfitz and M. Shearer, Berlin, Springer, 107-125, 1990.

[9] J. Li, T. Zhang, and S. Yang, The Two-Dimensional Riemann Problem in Gas Dynamics, Pitman Monographs and Surveys in Pure and Applied Mathematics, 98, Longman Scientific and Technical, 1998.

[10] W. B. Lindquist, The scalar Riemann problem in two spatial dimensions: Piecewise smoothness of solutions and its breakdown, SIAM J. Math. Anal. 17 (1986), no. 5, 1178-1197.

[11] _ Construction of solutions for two-dimensional Riemann problems, Comput. Math. Appl. Part A 12 (1986), no. 4-5, 615-630.

[12] T. P. Liu and J. Smoller, On the vacuum state for isentropic gas dynamic equations, Adv. in Appl. Math. 1 (1980), no. 4, 345-359.

[13] M. Nedeljkov, Shadow waves: entropies and interactions for delta and singular shocks, Arch. Ration. Mech. Anal. 197 (2010), no. 2, 487-537.

[14] M. Nedeljkov and M. Oberguggenberger, Interactions of delta shock waves in a strictly hyperbolic system of conservation laws, J. Math. Anal. Appl. 344 (2008), no. 2, 11431157.

[15] V. M. Shelkovich, Singular solutions of $\delta$ - and $\delta^{\prime}$-shock wave type of systems of conservation laws, and transport and concentration processes, Uspekhi Mat. Nauk 63 (2008), no. 3(381), 73-146; translation in Russian Math. Surveys 63 (2008), no. 3, 473-546.

[16] C. Shen and M. Sun, Formation of delta shocks and vacuum states in the vanishing pressure limit of Riemann solutions to the perturbed Aw-Rascle model, J. Differential Equations 249 (2010), no. 12, 3024-3051.

[17] Interactions of delta shock waves for the transport equations with split delta functions, J. Math. Anal. Appl. 351 (2009), no 2, 747-755.

[18] C. Shen, M. Sun, and Z. Wang, Global structure of Riemann solutions to a system of two-dimensional hyperbolic conservation laws, Nonlinear Anal. 74 (2011), no. 14, 4754-4770.

[19] W. Sheng and T. Zhang, The Riemann problem for the transportation equations in gas dynamics, Mem. Amer. Math. Soc. 137 (1999), no. 654, viii+77 pp. 
[20] W. Sun and W. Sheng, The non-selfsimilar Riemann problem for 2-D zero-pressure flow in gas dynamics, Chin. Ann. Math. Ser. B 28 (2007), no. 6, 701-708.

[21] D. Tan and T. Zhang, Two-dimensional Riemann problem for a hyperbolic system of nonlinear conservation laws, (I): Four-J cases; (II): Initial data consists of some rarefaction, J. Differential Equations 111 (1994), no. 2, 203-254; J. Differential Equations 111 (1994), no. 2, 255-283.

[22] D. Yoon and W. Hwang, Two-dimensional Riemann problem for Burgers equations, Bull. Korean Math. Soc. 45 (2008), no 1, 191-205.

[23] T. Zhang and Y. Zheng, Conjecture on the structure of solutions of the Riemann problem for two-dimensional gas dynamics systems, SIAM J. Math. Anal. 21 (1990), no. 3, 593630 .

[24] Y. Zheng, Systems of Conservation Laws, Birkhäuser Verlag, 2001.

School of Mathematics and Information

LUDONG UNIVERSITY

Yantai, Shandong Province 264025, P. R. China

E-mail address: smnwhy0350@163.com 